\title{
The microRNA, miR-29c, participates in muscle development through targeting the $Y Y 1$ gene and is associated with postmortem muscle pH in pigs
}

\author{
Weiya ZHANG', Wei WEI ${ }^{1,2}$, Yuanyuan $\mathrm{ZHAO}^{1}$, Shuhong $\mathrm{ZHAO}^{1}$, Xinyun LI (凶) ${ }^{1}$ \\ 1 Key Lab of Agricultural Animal Genetics, Breeding and Reproduction of Ministry of Education \& Key Laboratory of Swine Genetics and \\ Breeding of Ministry of Agriculture, Huazhong Agricultural University, Wuhan 430070, China \\ 2 College of Animal Science and Technology, Nanjing Agricultural University, Nanjing 210095, China
}

\begin{abstract}
Previous studies indicated that miR-29c is important for muscle development in mice and human, but its role in pigs is unknown. In this study, we detected the expression of miR-29c in Meishan longissimus lumborum (LL) muscle. The results showed that miR-29c was gradually upregulated during development of skeletal muscle in pig. Moreover, the expression of $Y Y 1$ and $A k t 3$ genes, which were confirmed to be targeted by miR-29c in mice, was decreased along with muscle development. Furthermore, the expression level of miR-29c was significantly higher in adult Meishan pigs than Large White pigs, while the expression of $Y Y 1$ and Akt3 genes was significantly lower in Meishan pigs. These results indicated that the expression pattern of miR-29c was opposite to that of $Y Y 1$ and Akt3 genes in pigs. Also, the luciferase assay indicated that miR-29s can target the $Y Y 1$ gene in pigs. In addition, we identified a $\mathrm{T}$ to $\mathrm{C}$ mutation in the primary transcript of miR-29c, which was associated with the postmortem muscle $\mathrm{pH}$ in pigs. Based on these results, we concluded that miR-29c is also important in skeletal muscle development of pigs.
\end{abstract}

Keywords pig, miR-29c, skeletal muscle, expression, SNP

\section{Introduction}

MicroRNAs are a class of small non-coding RNAs ${ }^{[1,2]}$ can inhibit target genes through binding with their $3^{\prime}$ untranslated regions $\left(3^{\prime} \text { UTR }\right)^{[3]}$. Previous studies showed

Received July 1, 2015; accepted November 13, 2015

Correspondence: hzlxy@163.com that microRNAs can participate in cell proliferation, differentiation and apoptosis ${ }^{[4-6]}$. The miR-29 family contains three members, miR-29a, miR-29b and miR$29 \mathrm{c}^{[7]}$, and they have similar expression patterns and biofunctions. MiR-29 can participate in many physiological and chemical processes. It has been reported that miR29a can inhibit apoptosis and protect the mitochondrial functions during forebrain ischemia through targeting the pro-apoptosis PUMA gene in astrocytes ${ }^{[8]}$. Also, many studies have shown that miR-29s can inhibit tissue fibrosis through downregulating collagen genes and inhibition of IGF-1 and PDGC growth factors ${ }^{[5,9,10]}$. Furthermore, miR29 s can restrain the activities of DNA methyltransferases and demethylases ${ }^{[11]}$, promote murine osteoclastogenesis $^{[12]}$ and inhibit tumorigenesis ${ }^{[13]}$.

MiR-29s are also important in skeletal muscle development. A recent study reported that miR-29 was downregulated in the dystrophic muscle and restoration of the expression of miR-29 in muscle tissue can improve dystrophy pathology by promoting regeneration and inhibiting of fibrogenesis ${ }^{[2]}$. Also, miR-29s can repress proliferation and promote differentiation of myoblasts in skeletal muscle development by targeting the Akt3 gene $^{[6]}$. Furthermore, they can regulate myogenesis via the NF$\mathrm{KB}-Y Y 1$-miR-29 signaling pathway in mic $\mathrm{e}^{[14]}$. Although some functions of miR-29s in muscle development have been reported in mice and human, this has not been studied in pigs. In this study, we focused on the role of miR-29c in muscle development in pigs.

\section{Materials and methods}

\subsection{Tissues and animals}

The longissimus lumborum (LL) muscle samples of

(C) The Author(s) 2015. Published by Higher Education Press. This is an open access article under the CC BY license (http://creativecommons.org/licenses/by/4.0) 
Meishan pigs at different stages, 50-day-old fetuses (E50d), 95-day-old fetuses (E95d) and adult stage (12 month), and Large White pigs at adult stage (12 month), were collected. The samples were stored at $-80^{\circ} \mathrm{C}$ until assayed by qPCR. A Large White population of 233 animals was selected for trait association analysis. All the pigs were slaughtered at about $90 \mathrm{~kg}$ and meat quality traits of intramuscular fat content (IMF) by Soxhlet extraction and $24 \mathrm{~h}$ postmortem muscle $\mathrm{pH}(\mathrm{pHu})$ determined by $\mathrm{pH}$ meter. In addition, muscle drip loss (DLS) and loin eye area were determined by methods described in previous studies $^{[1,15]}$.

\subsection{Cells and transfection}

PK-15 cells (a porcine kidney epithelial cell line) were cultured in high-glucose Dulbecco's modified Eagle's medium (DMEM) (Hyclone, Logan, UT, USA) with 12\% $(\mathrm{v} / \mathrm{v})$ fetal bovine serum. Cells were transferred to 24 well plates with growth medium, $24 \mathrm{~h}$ before transfection. Cells were transfected with miRNA mimics (GenePharma, Shanghai, China) and plasmid using the Lipofectamine 2000 transfection reagent (Invitrogen, Carlsbad, CA, USA). Opti-MEM I Reduced Serum Medium (Gibco, Grand Island, NY, USA) was used to dilute Lipofectamine 2000 and nucleic acids. Transfection procedure was performed follow the manufacturer's instructions.

\subsection{Cloning for dual-luciferase assay}

The psiCHECK-2 dual-luciferase reporter vector (Promega, Madison, WI, USA) housing the $3^{\prime}$ UTR of $Y Y 1$ gene was used to examine the effect of miR-29 on Renilla luciferase production. YY1 3' UTR was amplified with the use of forward primer $5^{\prime}$ CCGCTCGAGCTCTATC TTGCTCTGTAATCTCG 3' and reverse primer 5' ATAAGAATGCGGCCGCTCCAATTTCTGGGAGGCTCA3'. A 2-base substitution in the seed sequence of miR-29 was introduced to create mutant forms of miR-29 when synthesized. The miRNA mimics and 3' UTR dualluciferase vector were co-transfected into cells using Lipofectamine 2000 (Invitrogen). Cells were assayed with the Dual-Luciferase Reporter Assay System (Promega) $24 \mathrm{~h}$ after transfection.

\subsection{Quantitative PCR}

Total RNA (including miRNA) was extracted from tissues with TRIzol reagent (Invitrogen). Concentration and quality of RNA were assessed by the NanoDrop 2000 (Thermo, Waltham, MA, USA) and denatured gel electrophoresis. Reverse transcription was performed using Prime Script $^{\text {TM }}$ RT Reagent Kit with gDNA Eraser (TAKARA BIO INC, Otsu, Shiga, Japan) with miRNA specific primers added to initiate cDNA synthesis. The quantitative
PCR (qPCR) reaction was carried out in the LightCycler 480 II (Roche, Basel, Switzerland) system, and the reaction mixture used LightCycler 480 SYBR Green I Master (Roche) (Appendix A, Table S1).

\subsection{Genotyping}

One SNP under study was selected in the ssc-miR-29b-2/c cluster. The SNP was genotyped by a polymerase chain reaction-restriction fragment length polymorphism (PCRRFLP) method. The PCR product (752 bp) of the SNP was digested with $N c o$ I restriction enzyme (Fermentas, Life Sciences, USA) at $37^{\circ} \mathrm{C}$ overnight. This restriction enzyme recognizes the sequence T-C. The T-allele carrying the PCR product was cleaved once by the enzyme generating two fragments (631 and $121 \mathrm{bp}$ ). All digestion products were separated by agarose gel electrophoresis and the association analysis was performed by SAS program (Appendix A, Table S1).

\section{Results}

\subsection{MiR-29c can target $Y Y 1$ gene}

Previous studies have confirmed that $A K T 3$ and $Y Y 1$ are the target genes of miR-29 in mouse ${ }^{[6,16]}$. To test whether the YY1 gene was also targeted by miR-29c in pigs, the conservation of target site of the miR-29c in the $Y Y 1$ gene was analyzed. We found that it was completely conserved among human, mice and pig (Fig. 1a). Then we performed the luciferase activation assays. The fragment of the $3^{\prime} \mathrm{UTR}$ of the pig YY1 gene, which contains the binding site of miR-29s, was cloned into the luciferase vector. Subsequently, the interaction between miR-29 and 3'UTR of the YY1 gene was evaluated through luciferase activity analysis. The results showed that miR-29a, miR-29b and miR-29c could significantly inhibit the luciferase activity when the 3' UTR was inserted (Fig. 1b). Moreover, the mutant miR-29 with two nucleotides substituted in the seed sequence did not inhibit the luciferase activity (Fig. 1c). These results indicated that the $Y Y 1$ gene was also targeted by miR-29s in pigs.

\subsection{Differential expression analysis of miR-29c, $Y Y 1$ and Akt3}

The expression of miR-29c at E95d was 1.8 fold higher than that of E50d and it was sharply increased approximately 170 fold at the adult stage (Fig. 2a). In addition, the expression patterns of $Y Y 1$ and Akt3 genes were also measured. The results showed that expression of both was decreased during muscle development in Meishan pigs (Fig. 2b, Fig. 2c). Moreover, expression of miR-29c was significantly higher in Meishan than that in Large White at 
(a)

Position 764-770 of YY1 3' UTR:

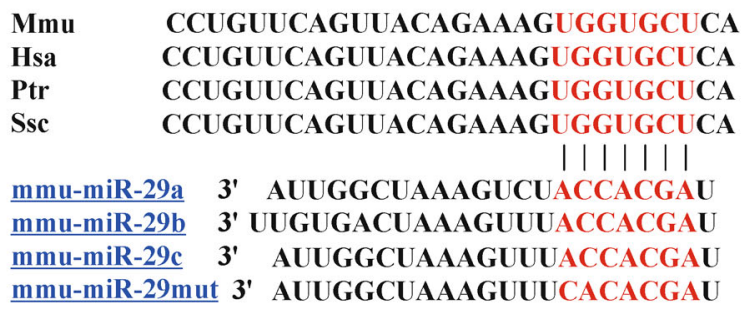

(b)

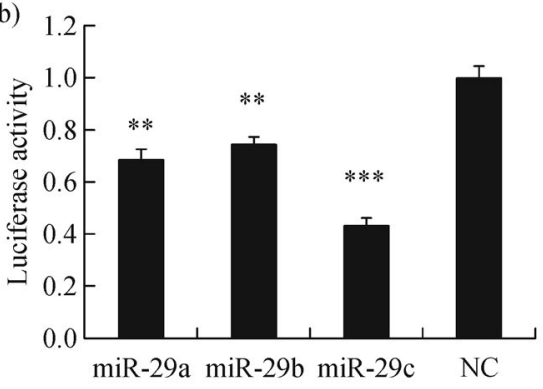

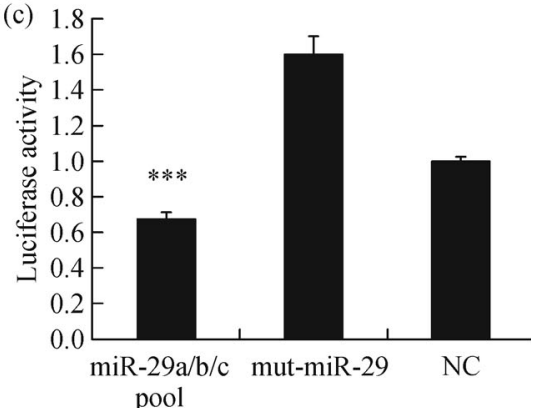

Fig. 1 Identification of the target genes of miR-29c. (a) The potential binding site of ssc-miR-29 in YY1 3' UTR, and the investigation of the conservation of this binding site in different species (http://www.targetscan.org); (b) identification of the target of $Y Y 1$ gene using dual luciferase reporter system. The 756 bp 3' UTR of YY1 gene was first inserted into the psiCHECK-2 luciferase vector, and then cotransfected into PK-15 cells together with miR-29a, miR-29b or miR-29c, and finally the luciferase activity was detected at 24 h posttransfection; (c) luciferase activity was detected when the mutant miR-29 (2 nt substitution was introduced into the seed sequence of miR29), pooled miR-29 or NC (negative control) were co-transfected with the luciferase vector inserted with the 765bp 3' UTR of YY1 gene. Results are shown as mean \pm S.E.M, means are of measurements from three independent individuals. ** Indicates a significant difference $(P<0.01) ; * * *$ indicates a significant difference $(P<0.001)$.

the adult stage (Fig. 3a). In contrast, the expression of $A k t 3$ and $Y Y 1$ was significantly lower in Meishan than in Large White (Fig. 3b, Fig. 3c). The expression patterns of YY1 and $A k t 3$ genes were opposite to that of miR-29c. These results also indicated that $Y Y 1$ and Akt 3 genes are targeted by miR-29c in pigs.

\subsection{The SNP in the ssc-miR-29b-2/c cluster influences $\mathrm{pHu}$}

We detected a C/T SNP in the ssc-miR-29b-2/c cluster at $443 \mathrm{nt}$ downstream of pre-miR-29b-2 and 33 nt upstream of pre-miR-29c, which caused a NcoI polymorphism (Fig. 4a, Fig. 4b). For genotyping, the 752 bp DNA fragment was amplified and the genotype was identified using PCR-RFLP analysis. As indicated, the CC genotype has one $752 \mathrm{bp}$ band; the TT genotype has 631 and $121 \mathrm{bp}$ bands, and the CT genotype has three bands (Fig. 4c). Furthermore, we found that the expression of miR-29c in the CC genotype was significant higher than in TT genotype (Fig. 4d).

The trait association of the Large White population showed that the polymorphism of this locus was significantly associated with the $\mathrm{pHu}$ trait. However, there was no significant association between this SNP and other muscle quality traits. According to our results, the mean value of $\mathrm{pHu}$ of $\mathrm{CC}$ individuals was significantly higher than TT individuals $(5.67 \pm 0.03$ vs $5.52 \pm 0.03$, $P<0.01$ ) (Table 1). This result showed that this polymorphism in miR-29c is important in determining the $\mathrm{pHu}$ trait of pig skeletal muscle.

\section{Discussion}

Previous studies indicated that miR-29s is important in myogenesis processes and it can target $Y Y 1$ and $A k t 3$ genes in mice ${ }^{[6,16]}$. In this study, we found that in pigs the expression of miR-29c was upregulated, while $Y Y 1$ and Akt3 were downregulated during muscle development. Also, through luciferase analysis we found that the porcine YY1 gene can be targeted by miR-29c. Therefore in pigs, miR-29c may also participate in muscle development through targeting $Y Y 1$ and $A k t 3$ genes. It has been reported that $Y Y 1$ and $A k t 3$ are important in myogenesis and muscle growth. In mice, our previous study found that Akt3 can promote the proliferation of myoblasts ${ }^{[6]}$. YY1 is a transcription factor containing a zinc finger DNA binding domain ${ }^{17]}$. It has been confirmed that $Y Y 1$ had negative 

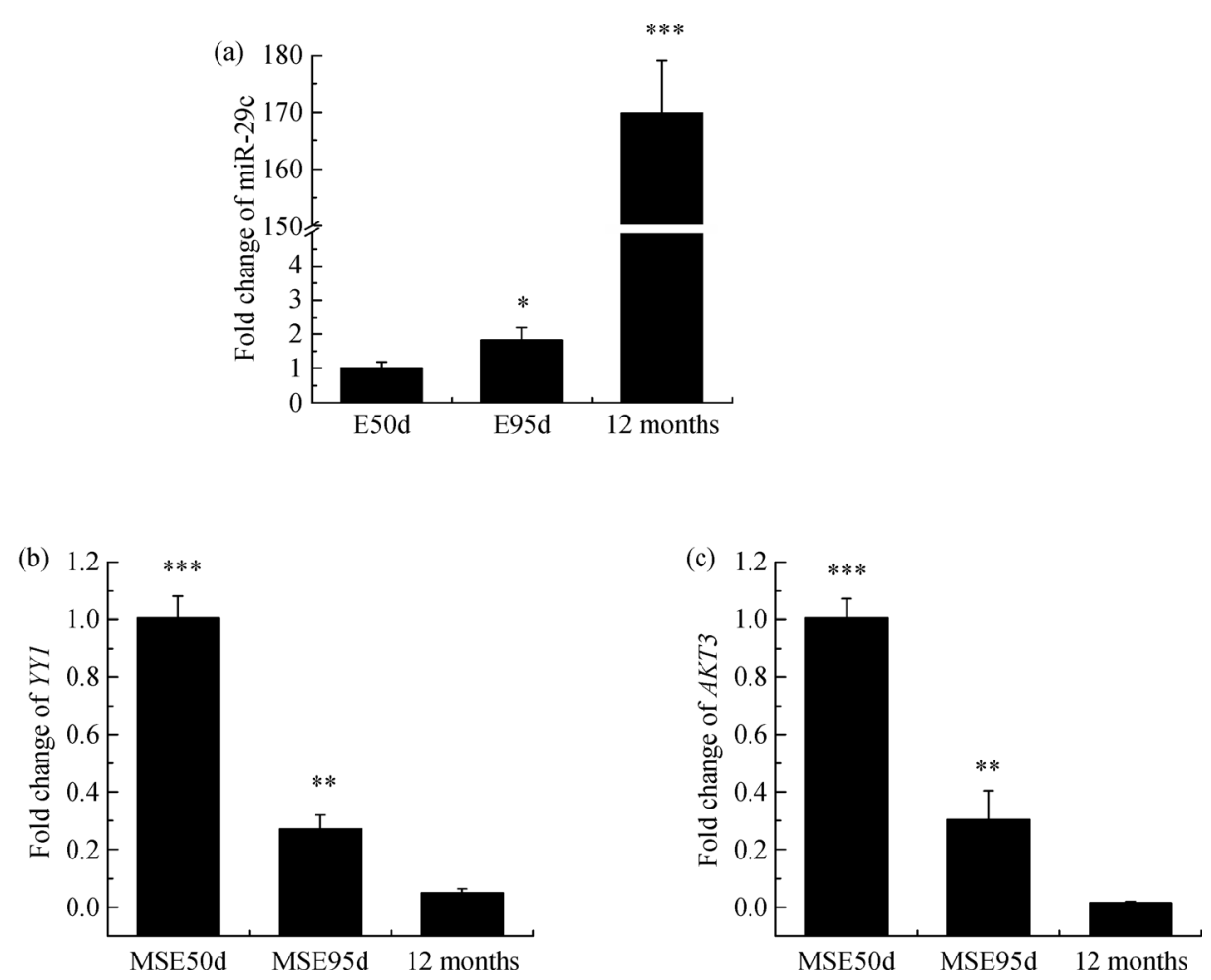

Fig. 2 Expression profiles of miR-29c, YY1 and Akt3 during muscle development. (a) The expression of miR-29c in LL muscle of Meishan pigs at E50d, E95d, and adult stage (12 months) was detected by qPCR; (b) the expression YY1 genes in LL muscle of Meishan at three stages was detected by qPCR; (c) the expression Akt3 genes in LL muscle of Meishan at three stages was detected by qPCR. The expression level of E50d was set as 1. Expression of miR-29c was normalized to U6. Expression of $A k t 3$ and $Y Y 1$ genes were normalized to $\beta$-Tubulin. Results are shown as mean \pm S.E.M., means are of three independent individuals. * Indicates a significant difference $(P<0.05) ; * *$ indicates a significant difference $(P<0.01) ; * * *$ indicates a significant difference $(P<0.001)$.

effects on myogenesis of muscle cells through inhibiting the expression of myogenic differentiation marker genes including $\alpha$-actin, Tnnc, Tnni2 and MyHC ${ }^{[18]}$. Moreover, YY1 can inhibit myogenesis through transcriptional regulation of non-coding RNAs including miR-1, miR29 and lncRNA Yam- ${ }^{[14,19]}$. Based on these results, we conclude that miR-29s can regulate skeletal muscle development through targeting $Y Y 1$ and $A k t 3$ genes in pigs.

In this study, we also found that miR-29 was associated with the $\mathrm{pH}$ value trait of pigs. Previous studies have shown that the $\mathrm{pH}$ value was associated with glycometabolism, and the concentration of glycogen in muscle tissue was positively correlated with its $\mathrm{pHu}$ value ${ }^{[20,21]}$. Also, previous studies indicated that both Akt3 and $Y Y 1$ played positive roles in glucose and energy metabolism of the muscle tissue. In mice, $Y Y 1$ knock out lead to hyperactivation of the insulin/IGF signaling, which can suppress the diabetic-like symptoms arising when treated with rapamycin. The YY1 protein can bind to the promoter regions of insulin/IGF and inhibit their transcription, which reduced the metabolism efficiency of blood glucose. Furthermore, YY1 can form a complex with mTOR and PGC-1 $\alpha$ to regulate mitochondrial genes expression and energy metabolism $^{[22]}$. A previous study indicated that $A k t 3$ also participated into glucose transport, and impaired Akt3 expression was related to insulin resistance of muscle tissue in humans ${ }^{[1]}$. In addition, glucose homeostasis was impaired in $A k t 2$ and $A k t 3$ genes double knockout mice and they displayed glucose and insulin intolerance ${ }^{[22-24]}$.

These studies indicated that $Y Y 1$ and $A K T 3$ are important in glucose and energy metabolism. Thus, miR-29c may affect the muscle $\mathrm{pHu}$ value through targeting Akt3 and YY1 genes.

\section{Conclusions}

In conclusion, miR-29c was upregulated, while $A k t 3$ and YY1 genes were downregulated, during muscle development of pigs. The miR-29c can participate in muscle development through targeting Akt 3 and $Y Y 1$ genes. A T to $\mathrm{C}$ mutation was detected in the miR-29c genomic sequence, which was association with $\mathrm{pHu}$ trait in pigs. Therefore, we conclude that miR-29 is important for the skeletal muscle development in pigs. 

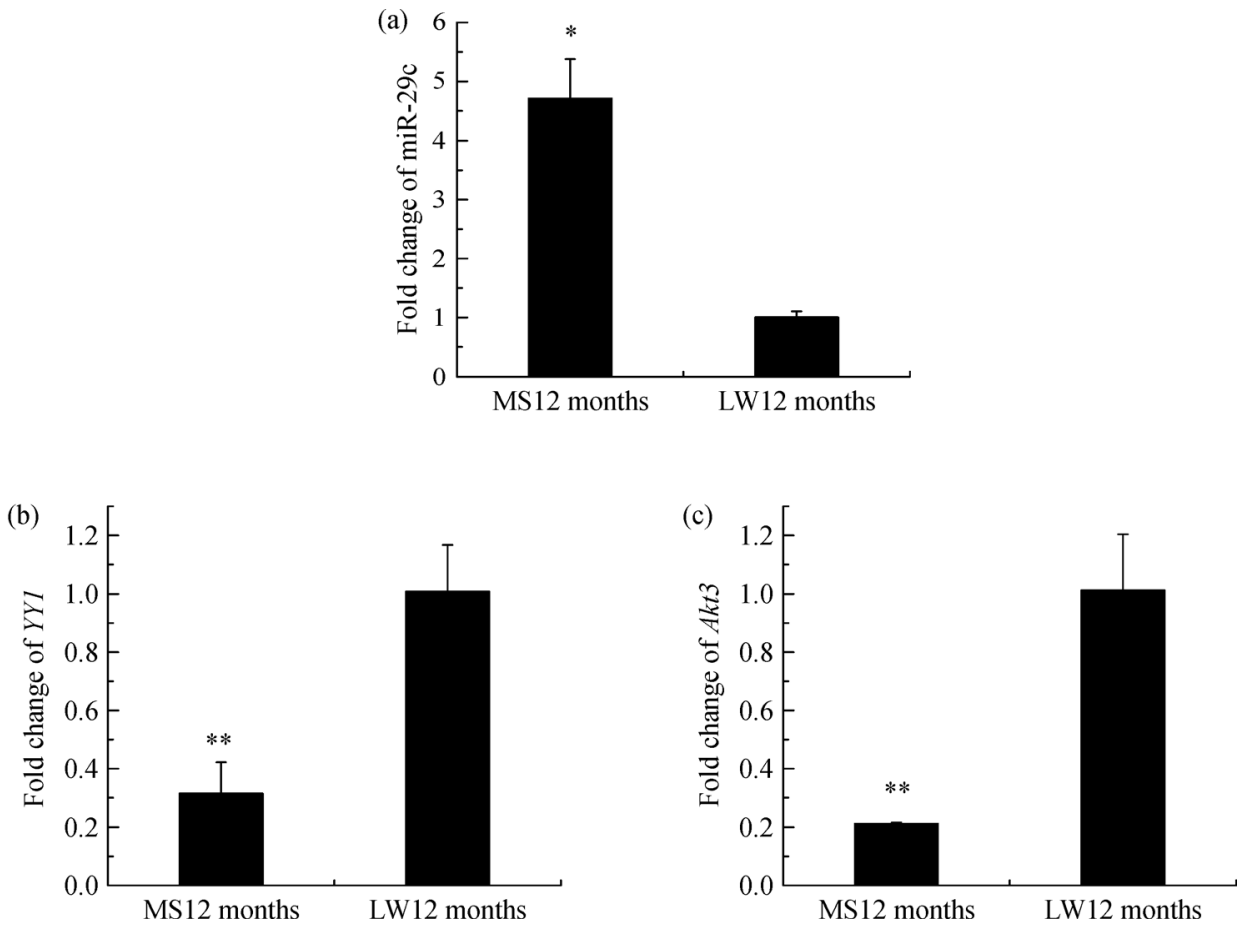

Fig. 3 Expression profiles of miR-29c, YY1 and Akt3 genes in different breeds. (a) The expression of miR-29c in LL muscle of Meishan and Large White at the adult stage (12 months); (b) the expression of YY1 genes in LL muscle of Meishan and Large White at the adult stage (12 months); (c) the expression of Akt3 genes in LL muscle of Meishan and Large White at the adult stage (12 months).The expression level of Large White was set as 1. Expression of miR-29c was normalized to U6. Expression of YY1 and Akt3 genes was normalized to $\beta$-Tubulin. Results are shown as mean \pm S.E.M., means are of three independent individuals. ${ }^{*}$ Indicates a significant difference $(P<0.05)$; ** indicates a significant difference $(P<0.01) ; * * *$ indicates a significant difference $(P<0.001)$.

(a)

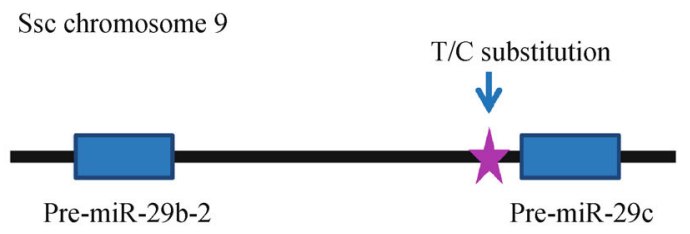

(c)

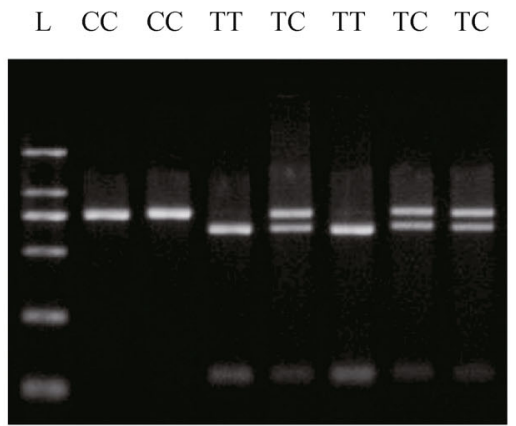

(b)

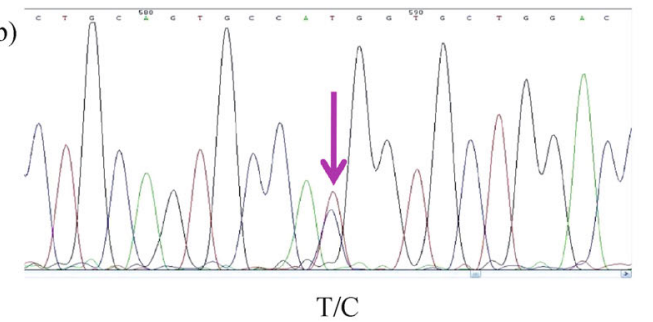

(d)

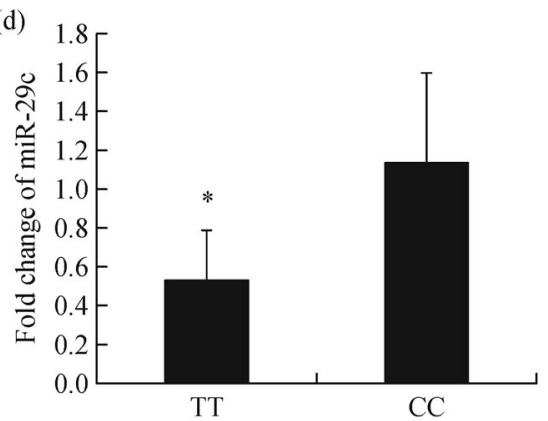

Fig. 4 SNP identification in the pre-miR-29b-2/c cluster. (a) The scheme of the SNP site in the pre-miR-29b-2/c cluster on SSC9; (b) the sequence of the T/C SNP. The SNP is labeled by an arrow; (c) the gel photo of the genotype digested using restriction enzyme NcoI. The CC homozygote had one band of $752 \mathrm{bp}$; the TT homozygote had two bands of 631 and $121 \mathrm{bp}$; TC heterozygote had bands of 752, 631 and $121 \mathrm{bp}$. L indicates the DNA ladder; (d) the expression level of miR-29c in TT and CC homozygote. The expression level of miR-29c in CC homozygote was set as 1.0 , expression of miR-29c was normalized to $U 6$. 
Table 1 Association study of the T/C SNP in the miR-29b-2/c cluster and meat quality traits

\begin{tabular}{|c|c|c|c|c|c|}
\hline Genotype & No. & Muscle $\mathrm{pHu}$ value & Muscle drip loss/\% & Intramuscular fat content $/ \%$ & Loin eye area $/ \mathrm{cm}^{2}$ \\
\hline $\mathrm{CC}$ & 46 & $5.67 \pm 0.03^{\mathrm{a}}$ & $1.60 \pm 0.21$ & $2.27 \pm 0.08$ & $46.17 \pm 0.91$ \\
\hline TC & 121 & $5.57 \pm 0.02^{\mathrm{b}}$ & $1.69 \pm 0.13$ & $2.23 \pm 0.05$ & $45.73 \pm 0.56$ \\
\hline TT & 65 & $5.53 \pm 0.03^{b}$ & $1.97 \pm 0.17$ & $2.20 \pm 0.07$ & $45.71 \pm 0.76$ \\
\hline \multicolumn{6}{|l|}{$P$ Value } \\
\hline CC-TC & & $0.0098 * *$ & 0.7329 & 0.6749 & 0.6827 \\
\hline CC-TT & & $0.0011 * *$ & 0.1743 & 0.4975 & 0.7009 \\
\hline TC-TT & & 0.2332 & 0.1873 & 0.7055 & 0.9845 \\
\hline
\end{tabular}

Note: Least square mean values with different letters are significantly different.

Acknowledgements This work was supported by the National Outstanding Youth Foundation of NSFC (31025026), National Basic Research Program of China (2012CB124702), and New Breed Creation Using Transgenic Methods (2014ZX0800950B).

Supplementary material The online version of this article at http:/ dx.doi.org/10.15302/J-FASE-2015075 contains supplementary material (Appendix A).

Compliance with ethics guidelines Weiya Zhang, Wei Wei, Yuanyuan Zhao, Shuhong Zhao, and Xinyun Li declare that they have no conflict of interest or financial conflicts to disclose.

All applicable institutional and national guidelines for the care and use of animals were followed.

\section{References}

1. Brozinick J T Jr, Roberts B R, Dohm G L. Defective signaling through Akt-2 and-3 but not Akt-1 in insulin-resistant human skeletal muscle: potential role in insulin resistance. Diabetes, 2003, 52(4): 935-941

2. Wang L, Zhou L, Jiang P, Lu L, Chen X, Lan H, Guttridge D C, Sun H, Wang H. Loss of miR-29 in myoblasts contributes to dystrophic muscle pathogenesis. Molecular Therapy, 2012, 20(6): 1222-1233

3. Bartel D P. MicroRNAs: genomics, biogenesis, mechanism, and function. Cell, 2004, 116(2): 281-297

4. Tiao M M, Wang F S, Huang L T, Chuang J H, Kuo H C, Yang Y L, Huang Y H. MicroRNA-29a protects against acute liver injury in a mouse model of obstructive jaundice via inhibition of the extrinsic apoptosis pathway. Apoptosis, 2012, 19(1): 30-41

5. Kwiecinski M, Elfimova N, Noetel A, Töx U, Steffen H M, Hacker U, Nischt R, Dienes H P, Odenthal M. Expression of plateletderived growth factor-C and insulin-like growth factor I in hepatic stellate cells is inhibited by miR-29. Laboratory Investigation, 2012, 92(7): 978-987

6. Wei W, He H B, Zhang W Y, Zhang H X, Bai J B, Liu H Z, Cao J H, Chang K C, Li X Y, Zhao S H. MiR-29 targets Akt3 to reduce proliferation and facilitate differentiation of myoblasts in skeletal muscle development. Cell Death \& Disease, 2013, 4(6): e668

7. Kriegel A J, Liu Y, Fang Y, Ding X, Liang M. The miR-29 family: genomics, cell biology, and relevance to renal and cardiovascular injury. Physiological Genomics, 2012, 44(4): 237-244

8. Ouyang Y B, Xu L, Lu Y, Sun X, Yue S, Xiong X X, Giffard R G. Astrocyte-enriched miR-29a targets PUMA and reduces neuronal vulnerability to forebrain ischemia. Wiley Periodicals, 2013, 61: 1784-1794

9. Nijhuis A, Biancheri P, Lewi A, Bishop C L, Giuffrida P, Chan C, Feakins R, Poulsom R, Di Sabatino A, Corazza G R, MacDonald T T, Lindsay J O, Silver A R. In Crohn's disease fibrosis reduced expression of the miR-29 family enhances collagen expression in intestinal fibroblasts. Clinical Science, 2014, 127(5): 341-350

10. Bandyopadhyay S, Friedman R C, Marquez R T, Keck K, Kong B, Icardi M S, Brown K E, Burge C B, Schmidt W N, Wang Y, McCaffrey A P. Hepatitis $C$ virus infection and hepatic stellate cell activation downregulate miR-29: miR-29 overexpression reduces hepatitis C viral abundance in culture. Journal of Infectious Diseases, 2011, 203(12): 1753-1762

11. Morita S, Horii T, Kimura M, Ochiya T, Tajima S, Hatada I. MiR-29 represses the activities of DNA methyltransferases and DNA demethylases. International Journal of Molecular Sciences, 2013, 14(7): 14647-14658

12. Franceschetti T, Kessler C B, Lee S K, Delany A M. miR-29 promotes murine osteoclastogenesis by regulating osteoclast commitment and migration. Journal of Biological Chemistry, 2013, 288(46): 33347-33360

13. Wang Y, Zhang X, Li H, Yu J, Ren X. The role of miRNA-29 family in cancer. European Journal of Cell Biology, 2013, 92(3): 123-128

14. Wang H, Garzon R, Sun H, Ladner K J, Singh R, Dahlman J, Cheng A, Hall B M, Qualman S J, Chandler D S, Croce C M, Guttridge D C. NF-kB-YY1-miR-29 regulatory circuitry in skeletal myogenesis and rhabdomyosarcoma. Cancer Cell, 2008, 14(5): 369-381

15. CaLLara F R, Moi M, dos Santos Luan S. Carcass characteristics and qualitative attributes of pork from immunocastrated animals. Asian-Australas Journal of Animal Science, 2013 , 26(11):16301636

16. Zhou L, Wang L, Lu L, Jiang P, Sun H, Wang H. A novel target of microRNA-29, Ring1 and YY1-binding protein (Rybp), negatively regulates skeletal myogenesis. Journal of Biological Chemistry, 2012, 287(30): 25255-25265

17. Kalenik J L, Chen D, Bradley M E. Yeast two-hybrid cloning of a novel zinc finger protein that interacts with the multifunctional transcription factor YY1. Nucleic Acids Research, 1997, 25(4): 843849

18. Shi Y, Seto E, Chang L S, Shenk T. Transcriptional repression by YY1, a human GLI-Krüippel-related protein, and relief of repression by adenovirus E1A protein. Cell, 1991, 67(2): 377-388

19. Lu L, Zhou L, Chen E Z, Sun K, Jiang P, Wang L, Su X, Sun H, 
Wang H. A Novel YY1-miR-1 regulatory circuit in skeletal myogenesis revealed by genome-wide prediction of YY1-miRNA network. PLoS ONE, 2012, 7(2): e27596

20. Monin G, Sellier P. Pork of low technological quality with a normal rate of muscle $\mathrm{pH}$ fall in the immediate post-mortem period: the case of the Hampshire breed. Meat Science, 1985, 13(1): 49-63

21. Sterten H, Oksbjerg N, Frøystein T, EkkerA S, Kjos N P. Effects of fasting prior to slaughter on $\mathrm{pH}$ development and energy metabolism post-mortem in M. longissimus dorsi of pigs. Meat Science, 2010, 84(1): 93-100

22. Cunningham J T, Rodgers J T, Arlow D H, Vazquez F, Mootha V K, Puigserver P. mTOR controls mitochondrial oxidative function through a YY1-PGC-1alpha transcriptional complex. Nature, 2007, 450(7170): 736-740

23. Dummler B, Tschopp O, Hynx D, Yang Z Z, Dirnhofer S, Hemmings B A. Life with a single Isoform of Akt: mice lacking $\mathrm{Akt} 2$ and Akt3 are viable but display impaired glucose homeostasis and growth deficiencies. Molecular and Cellular Biology, 2006, 26 (21): 8042-8051

24. Blattler S M, Cunningham J T, Verdeguer F, Chim H, Haas W, Liu H, Romanino K, Ruegg M A, Gygi S P, Shi Y, Puigserver P. Yin Yang 1 deficiency in skeletal muscle protects against rapamycininduced diabetic-like symptoms through activation of insulin/IGF signaling. Cell Metabolism, 2012, 15(4): 505-517 